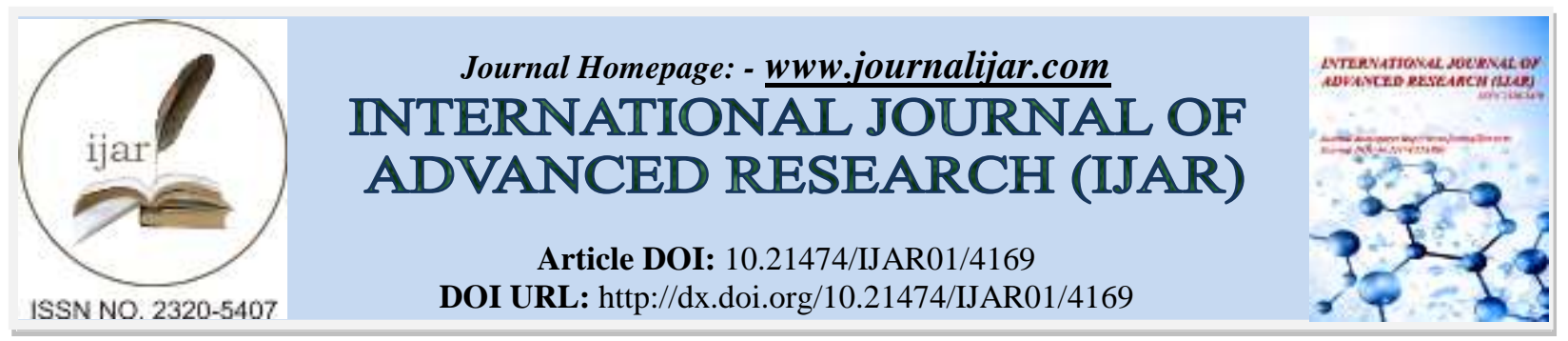

RESEARCH ARTICLE

\title{
REVIEW ON LOCAL BINARY PATTERN (LBP) TEXTURE DESCRIPTOR AND ITS VARIANTS.
}

\section{Sajida Parveen ${ }^{1, *}$, Nadeem Naeem ${ }^{2}$ and Jherna Devi ${ }^{3}$}

1. Department of Computer Systems Engineering, Quaid-e-Awam univertersity of Engineering, Science and Technology, Nawabshah, Pakistan.

2. Department of Electronic Engineering, Quaid-e-Awam univertersity of Engineering, Science and Technology, Nawabshah, Pakistan.

3. Department of Information Technology, Quaid-e-Awam univertersity of Engineering, Science and Technology, Nawabshah, Pakistan.

\section{Manuscript Info}

Manuscript History

Received: 07 March 2017

Final Accepted: 09 April 2017

Published: May 2017

Key words:-

Texture descriptors; Local Binary

Pattern; Feature descriptor, extended version of LBP

\section{Abstract}

Local binary pattern and its variants have been extensively used in the numerous applications of various fields such as computer vision, pattern recognition, and texture analysis and etc. Comprehensive review based on the study of Local Binary Pattern and its variants is providing in this article. The detailed stat-of-art feature descriptors which are mostly used for number of application to analyze the textural properties have been discussed along with their pros and cons. The conclusion is made on the basis of their limitations and highlighted the gapes of recent variants of LBP texture descriptors.

Copy Right, IJAR, 2016,. All rights reserved.

\section{Introduction:-}

The important characteristic of visual appearance of any object surface is considered as a Texture. Textures can be defined as very small particles like sand or huge elements like stars in Milky Way. All the objects the universe consisted on different texture and is different from each other. While, different degree of visual attention, distance, scales, occlusion, background, environmental condition and viewing point can create different interpretations [1,2].

The texture characteristics of digital images can be defined as variations in spatial structure (patterns) and contrast (strength of pattern

Particularly in the field of computer vision, the appearance of the digital image texture is viewed with respect to the pixels of image. The Texture analysis is used to considere the significant change or variation in the pixel intensity and non-homogeneity between nearby pixels.

In real world scenario, there is a great variability and challenging problem related to the number of applications. For expecting a good final result of any texture analysis based method or algorithm which provides the necessary information in the features and should tolerate the variations such as illumination, rotations and scales as well for the particular application, the good image descriptors are essential.

\section{Corresponding Author:- Sajida Parveen.}

Address:- Department of Computer Systems Engineering, Quaid-e-Awam univertersity of Engineering, 
For selecting the descriptor to exploit textures in various field of image processing, it is important that the feature measures should be capable enough in detecting different texture properties and also consider the issues related to the computational complexity to make the method realistic in use and take reasonable classification time.

\section{Literature Review:-}

Basically the methods of textures analysis are categories into four types [3]: (1) Structural, (2) Statistical, (3) Model based and (4) Transforms.

\section{Structural:-}

These methods represent texture by primitive patterns which are regular in appearance and systematically located on the surface.

\section{Statistical:-}

In contrast to the structural methods, statistical analysis based method do not consist on regular or systematical structure. Instead they represent the texture by non-deterministic properties of image pixels and regions which are usually natural and consist on randomly distributed surface elements.

\section{Model:-}

The model based texture analysis methods are combination of fractal and stochastic models.

\section{Transforms: -}

In transform based methods the image is represented according to the coordinate system. For example Fourier, Wavelet and Gabor transforms interpreted the closely related texture characteristics from their coordinate system.

Numerous local texture descriptors have been introduced in the field of texture analysis. The recent advances in newly proposed texture descriptors promise a significant progress in trends of texture analysis by means of their high discriminative properties and computational efficiency. From the state-of-art, it is clear that LBP and its variants outperformed then other reported descriptors in the field of texture analysis based applications [4].

Basically the texture of an image was decomposes into set of useful units [5]. In this method mapping is done via local 8 surrounding neighborhood pixels and encoded the generated unit of texture in one of three values i.e 0,1 or 2. The texture unit number in this technique is computed as follow:

$$
\begin{aligned}
& T U N=\sum_{p=0}^{n} e_{p} \quad L^{p-1} \\
& e_{p}=\left\{\begin{array}{lll}
0, & \text { if } & \left(w_{p}>w_{c}\right) \\
1, & \text { if } & \left(w_{p}=w_{c}\right) \\
2, & \text { if } & \left(w_{p}<w_{c}\right)
\end{array}\right.
\end{aligned}
$$

Where $e_{p}$ is calculated with number of levels $\mathrm{L}=3$, with $\mathrm{p}=1, \ldots, 8$ by comparing the intensity value of a center pixel $w_{c}$ with a neighboring pixel $w_{p}$ in either a clockwise or counter clockwise direction. The number of features can be defined by $3^{8}=6561$. Then texture of an image can be described with a histogram of these values.

Later on in 1994 the levels of operator reduced from $\mathrm{L}=3$ to $\mathrm{L}=2$, and comparisons were performed by boolean operation in first LBP operator [6]. The intensity values were calculated by comparing neighborhood pixel with central pixel value, moving from top to bottom and left to right as in following expression:

$e_{p}= \begin{cases}1, & \left(w_{p}>w_{c}\right) \\ 0, & \text { otherwise }\end{cases}$

With $L=2$, the size of feature vector is reduces and make it compactable with values $2^{8}$. However, this compactness comes at a cost: these descriptors are more sensitive to noise, especially in the near-uniform regions of an image $[7,8]$. 


\section{Local Binary Pattern (LBP):-}

LBP proposed by Ojala et. Al. [6], it is very famous because of its simple representation, efficient calculation, low in computational complexity and fine detail coding ability. LBP descriptor is the combination of statistical and structural based Model. The other very powerful extensions of LBP with rotation invariance multi-resolution also have been developed by Ojala et. al. [7].

Original LBP operator is rotation invariant to gray scale texture [8]. The extraction of LBP operator comprises two main steps: one is thresholding and second is encoding step. The thresholding step extract the information about the local binary difference (1or 0) by comparing the circular neighbor pixels value with central pixel in a patch of the image.

$s(x)=\left(w_{c}, w_{0}-w_{c}, w_{1}-w_{c}, \ldots w_{p-1}-w_{c}\right)$

Where $w_{c}$ and $w_{p}$ are defined in equation (2) and sampling number $p(p=0, \ldots, p-1)$ in equation (3).

$$
s(x)=\left\{\begin{array}{llc}
1 & \text { if } \quad x \geq 1 \\
0 & \text { othrewise }
\end{array}\right.
$$

Then in the second encoding step, the obtained binary numbers from first step are converted into decimal number as shown in Figure1.

The first version of LBP operator is work with $3 \times 3$ patch of an image but later on this patch size have no fix rule for using sampling points in the circle in other extension version [8]. Instead of using $3 \times 3$ size of the patch, it can be change with the values of $P, R$ such as $(8,1),(16,2),(8,2)$ and so on. The mathematical equation can be defined as follow:

$$
L B P_{P, R}=\sum_{p=0}^{P-1} s\left(w_{p}-w_{c}\right) \times 2^{p}
$$

Where $w_{c}, w_{p}$ and $p$ are already defined in equation (2),(3) and (4). And $\mathrm{P}, \mathrm{R}$ is the number of neighbors or samples and radius of the circle respectively.

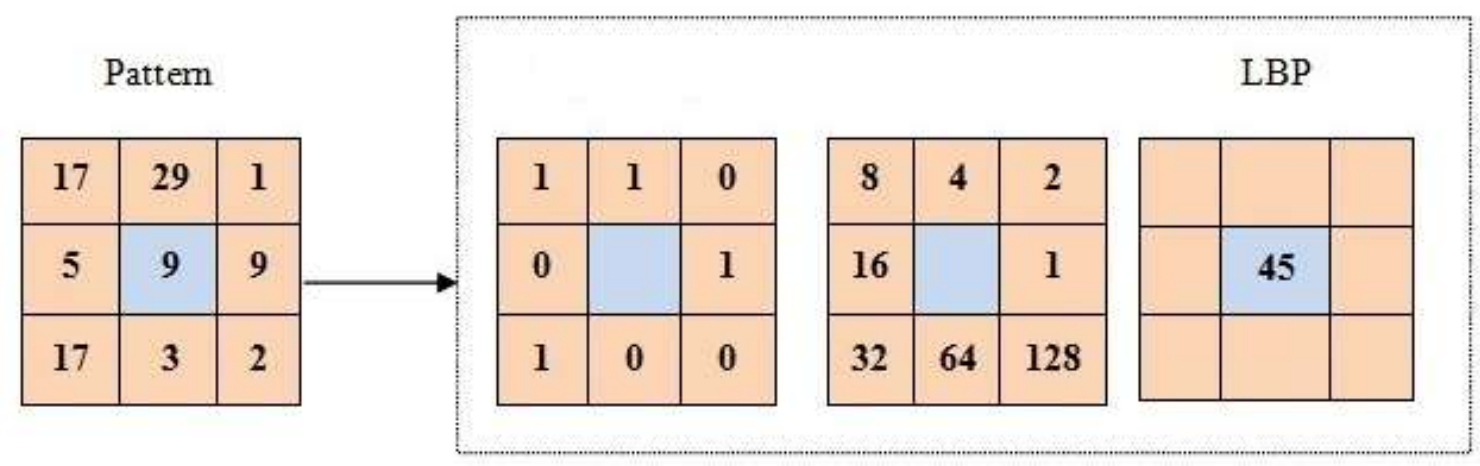

Fig 1:- LBP calculation on $3 \times 3$ patch on image

Another variation in original operator seems in the form of uniform patterns [9]. A Uniform pattern can be denoted with ' $U$ ' and defined as its uniformity (number of transactions from 0 to 1 or from 1 to 0 bitwise) measure is at most 2. There are main reasons for considering only uniform pattern, one that in real world scenario most of the texture have uniform in nature and themselves are more stable i.e. less affected by noise and another reason is, because only consider uniform pattern so the minimum samples of LBP are reliable to recognized the texture pattern [10].

\section{Complete Local Binary Pattern (CLBP):-}

Typical Local binary Pattern operator only considered the local features of an image and global features are neglected. To improve the performance of LBP by considering global thresholding and a new approach named as Complete Local Binary Pattern is proposed by [11]. The image descriptor is split into two complementary components: sign and magnitude component as shown in equation below: 
$s_{p}=s\left(w_{p}-w_{c}\right), m_{p}=\left|w_{p}-w_{c}\right|$

Where $s_{p}$ is sign and $m_{p}$ is used to build the magnitude component of CLBP_S and CLBP_M respectively. CLBP_S is the same to traditional LBP operator while in CLBP_M the local variance of the magnitude is calculated. These two components can be described mathematically as follow:

$$
\begin{aligned}
& C L B P_{-} S_{P, R}=\sum_{p=0}^{P-1} 2^{p} s\left(w_{p}-w_{c}\right), \\
& s_{p}= \begin{cases}1, & w_{p} \geq w_{c}, \\
0, & w_{p}<w_{c},\end{cases} \\
& C L B P_{-} M_{P, R}=\sum_{p=0}^{P-1} 2^{p} t\left(m_{p}, c\right), \\
& t\left(m_{p}, c\right)= \begin{cases}1, & \left|w_{p}-w_{c}\right| \geq c, \\
0, & \left|w_{p}-w_{c}\right|<c,\end{cases}
\end{aligned}
$$

Where $w_{c}, w_{p}$ and $p$ are defined before in equation (2) and $c$ in equation (10) denoted the mean value of magnitude component in whole image. The authors calculated the global features by using center pixel as threshold and coded into binary format. This operator called CLBP_Center. The CLBP_C can be defined mathematically in the following expression:

$$
C L B P_{-} C_{P, R}=t\left(w_{c}, c_{i}\right) \text {, }
$$

Where $w_{c}$ is the center pixels value and $c_{i}$ is the average gray level of the whole image.

The calculated features from these three operators can be combined into joint histogram for final texture classification.

\section{Local Ternary Pattern Techniques:-}

The result of LBP algorithm is grounded on $\mathrm{L}=2$ means binary number operation by specifying the center pixel intensity value as a threshold. As the threshold value is center pixel, this is the main reason that LBP descriptor is more sensitive to noise specially for smooth regions of the images. To overcome this noise sensitivity problem an interesting abstraction of LBP known as is Local Ternary Pattern proposed by Tan et al [12].

In LTP, the three quantization levels were used to encode the difference between central pixel and its neighbor to a threshold. Generally the threshold value set to be manually, and its value depends in order to attain the best performance in specific problems and applications. The threshold equation for ternary operation is given below.

$$
s(x)=\left\{\begin{array}{clc}
1 & \text { if } & w_{p}>w_{c}+t \\
0 & \text { if } & w_{c}-t \leq w_{p} \leq w_{c}+t \\
-1 & \text { if } & w_{p}<w_{c}-t
\end{array}\right.
$$

Where $w_{c}$ and $w_{p}$ are defined in equation (2),(3) . $t$ is a threshold value that controls the sensitivity of the ternary pattern against noise. To reduce the size of ternary code, the scheme is spited into two binary threshold function: upper and lower function. The upper pattern is denoted with $h_{i}$ and can be computed as:

$h_{i}=\left\{\begin{array}{cc}1 & \text { if } \quad a_{i}=1 \\ 0 & \text { othrewise }\end{array},(1 \leq i \leq P)\right.$ 
The binary threshold function $l i$ for the lower pattern is calculated:

$l_{i}=\left\{\begin{array}{cc}1 & \text { if } \quad a_{i}=-1 \\ 0 & \text { otherwise }\end{array},(1 \leq i \leq P)\right.$

The weighted sum function for upper pattern can be expressed as:

$$
L T P h_{P, R}=\sum_{i=1}^{P} h_{i} \times 2^{i-1},\left(h_{i} \in\{0,1\}\right)
$$

The weighted sum function for lower pattern can be expressed as:

$$
\operatorname{LTPl}_{P, R}=\sum_{i=1}^{P} l_{i} \times 2^{i-1},\left(l_{i} \in\{0,1\}\right)
$$

The final LTP operator is constructed with the concatenation of the lower and upper patterns. The computational process of LTP on $3 \times 3$ image patch is represented in Figure 2.

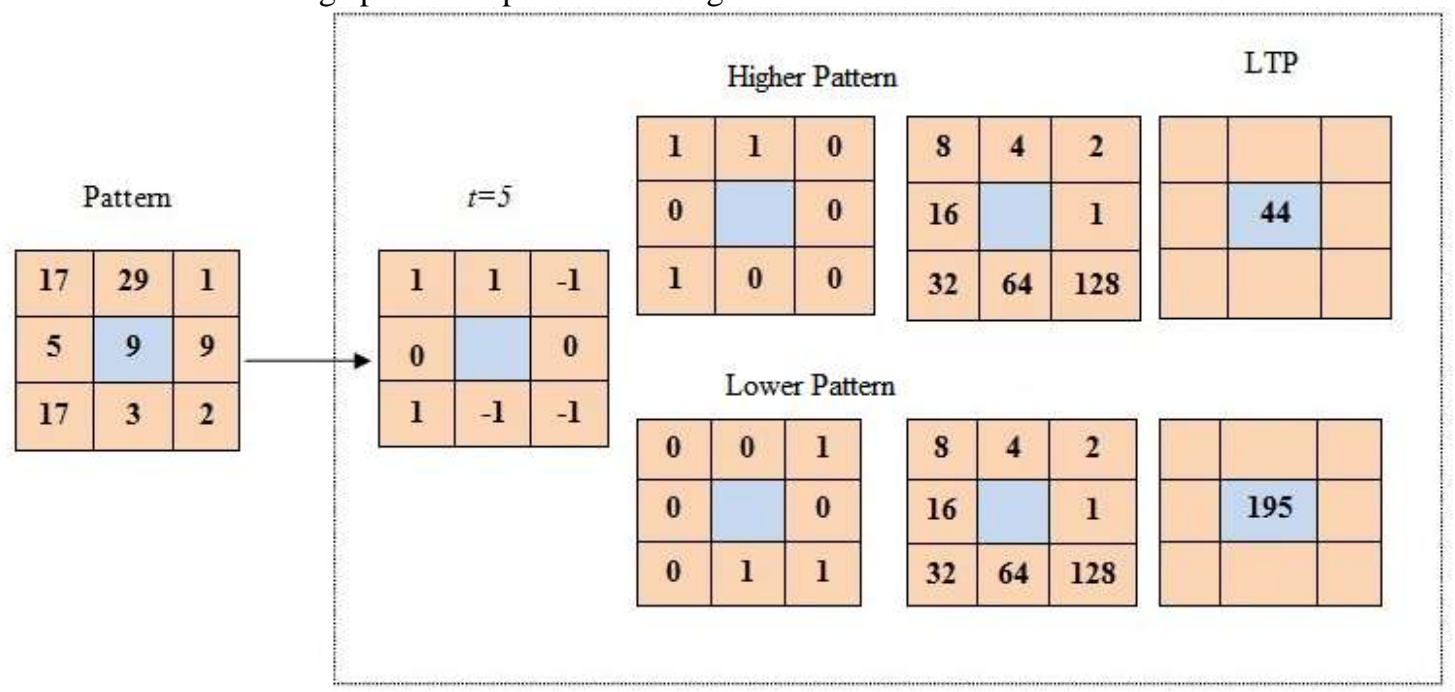

Fig 2:- Calculation of LTP operators. The obtained ternary pattern is further coded into higher and lower binary code in the LTP

\section{Complete Local Ternary Pattern (CLTP):-}

Recently, a new diversion was proposed by [13] in which the authors introduce the global features in LTP [12] as similar to CLBP [11] and named as Complete Local Ternary Pattern CLTP. The local difference of an image was further decomposes into two sign and two magnitude components. The two decomposition of each component can be denoted with upper and lower patterns and mathematically can be described as follows:

$$
\begin{aligned}
& s_{p}^{\text {upper }}=s\left(w_{p}-\left(w_{c}+t\right)\right), \quad s_{p}^{\text {lower }}=s\left(w_{p}-\left(w_{c}-t\right)\right) \\
& m_{p}^{\text {upper }}=\left|w_{p}-\left(w_{c}+t\right)\right|, m_{p}^{\text {lower }}=\left|w_{p}-\left(w_{c}-t\right)\right|, \\
& \left.C L T P_{-} S_{P, R}^{\text {upper }}=\sum_{p=0}^{P-1} 2^{p} s\left(w_{p}-w_{c}+t\right)\right), \\
& s_{p}^{\text {upper }}= \begin{cases}1, & w_{p} \geq w_{c}+t \\
0, & \text { otherwise },\end{cases}
\end{aligned}
$$




$$
\begin{aligned}
& \text { CLTP_ } S_{P, R}^{\text {lower }}=\sum_{p=0}^{P-1} 2^{p} s\left(i_{p}-\left(i_{c}-t\right)\right), \\
& s_{p}^{\text {lower }}= \begin{cases}1, & i_{p}<i_{c}-t \\
0, & \text { otherwise },\end{cases}
\end{aligned}
$$

Where $w_{c}, w_{p}, p$ and $t$ are already defined in equation (2) and (13). The final CLTP_S operator can be constructed by concatenating upper and lower components as follow:

$$
C T L P_{-} S_{P, R}=\left[C L T P_{-} S_{P, R}^{\text {upper }} \quad C L T P_{-} S_{P, R}^{u p p e r}\right]
$$

In the similar way of sign component, magnitude component of the CLTP can be built by using upper and lower pattern and mathematically can be defined in the following expressions:

$$
\begin{aligned}
& C L T P_{-} M_{P, R}^{\text {upper }}=\sum_{p=0}^{P-1} 2^{p} t\left(m_{p}^{\text {upper }}, c\right), \\
& t\left(m_{p}^{\text {upper }}, c\right)= \begin{cases}1, & \left|w_{p}-\left(w_{c}+t\right)\right| \geq c, \\
0, & \left|w_{p}-\left(w_{c}+t\right)\right|<c,\end{cases} \\
& C L T P_{-} M_{P, R}^{\text {lower }}=\sum_{p=0}^{P-1} 2^{p} t\left(m_{p}^{\text {lower }}, c\right), \\
& t\left(m_{p}^{\text {lower }}, c\right)= \begin{cases}1, & \left|w_{p}-\left(w_{c}-t\right)\right| \geq c, \\
0, & \left|w_{p}-\left(w_{c}-t\right)\right|<c,\end{cases}
\end{aligned}
$$

Where $w_{c}, w_{p}, p$ and $t$ are already defined in equation (2) and (13) and $c$ is defined already in equation (10). Similar to the LTP and CLTP_S, upper and lower patter concatenated to built the CLTP_M operator as follow:

$$
C L T P_{-} M_{P, R}=\left[C L T P_{-} M_{P, R}^{\text {upper }} \quad C_{\text {CTP }} M_{P, R}^{\text {lower }}\right] \text {, }
$$

Moreover, center thresholding code for upper and lower pattern can be constructed as follow:

$$
\begin{aligned}
& C L T P_{-} C_{P, R}^{\text {upper }}=t\left(w_{c}^{\text {upper }}, c_{i}\right), \\
& C L T C_{-} C_{P, R}^{\text {lower }}=t\left(w_{c}^{\text {lower }}, c_{i}\right),
\end{aligned}
$$

Where $w_{c}^{\text {upper }}=w_{c}+t, w_{c}^{\text {lower }}=w_{c}-t$ and $c_{i}$ is defined before in equation (12). The final CLTP operator combined into joint histogram.

\section{Improved Local Ternary Pattern (ILTP):-}

The modified version of LTP that is known as Improved Local Ternary Patter (ILTP) [14] was introduced to reduce the sensitivity of noise in central pixels because it is used as threshold to encode. The method is based on replacement of central pixel value with the average value of a patch or region. The mathematical representation of this operator is same as of LTP except center pixel value which is shown in the following equation.

$$
I L T P_{P, R}=\sum_{p=0}^{P-1} 2^{p} s\left(w_{p}-a\right)
$$

Where all the parameters are already defined in equation (6) and $a$ is the average value of the region. 


\section{Local Adaptive Ternary Pattern (LATP):-}

In original LTP operator the value of threshold need to be set manually. Though, it is very critical task to set a threshold value for calculating all local patches in order to reduce the sensitivity to noise for different applications. Local Adaptive Ternary Pattern [15] was proposed, in which the threshold value is set with automatic adaptive procedure by using local statistics as a mean value and the standard deviation. Equation for LATP is expressed as follow:

$$
s(x)=\left\{\begin{array}{clc}
1 & \text { if } & w_{p} \geq(\alpha+k \beta) \\
0 & \text { if } & \left|w_{p}\right|<(\alpha+k \beta) \\
-1 & \text { if } & w_{p} \leq(\alpha+k \beta)
\end{array}\right.
$$

Where $w_{p}$ is defined in equation (3), $\alpha$ is the mean of the local patch or region, standard deviation is denoted with $\beta$ and $k$ is a constant value.

\section{New techniques based on Weber's Law: ELTP and DLTP:-}

Recently, in order to deal with the problem of setting proper threshold value of Ternary coding for the region, some modified approaches come up with a new idea to set the threshold value inspired from the Weber's Law. According to the Weber's law, the ratio of the increment threshold to the background intensity is a constant. The relationship can be expressed as:

$$
\frac{\Delta I}{I}=k
$$

Where $\Delta I$ is the increment threshold (just noticeable difference for discrimination); $I$ represents the initial stimulus intensity and $k$ is the constant which signifies that the proportion on the left side of the equation remains constant despite variations in the $I$ term.

The first approach proposed in January 2014 known as Dynamic Local Ternary Pattern [16] and the second approach called as Enhanced Local Ternary Pattern [17] proposed in July 2014. According to [16], which relies on Weber's law for tuning of the threshold value, a dynamic process was adopted to assign different threshold values instead of choosing a fix threshold value. By the local statistics of the pattern, threshold equation can be expressed as;

$$
t=w_{c} \times \gamma
$$

Where $\gamma$ is the scaling factor as constant in Weber's law, $w_{c}$ is the intensity value of center pixel of the patch. This $t$ is a threshold value which can be used to generate dynamic threshold value for every local region and then can be code in ternary quantization level as in original LTP code as shown below:

$s(x)=\left\{\begin{array}{ccc}1 & \text { if } & w_{p}>w_{c}+t \\ -1 & \text { if } & w_{p} \leq w_{c}-t \\ 0 & \text { otherwise } & \end{array}\right.$

Where $w_{p}$ is defined in equation (3), and $w_{c}$ and $t$ is defined in equation (35).

The Local Binary Pattern (LBP) and its extended versions are summarized in Figure 3. 


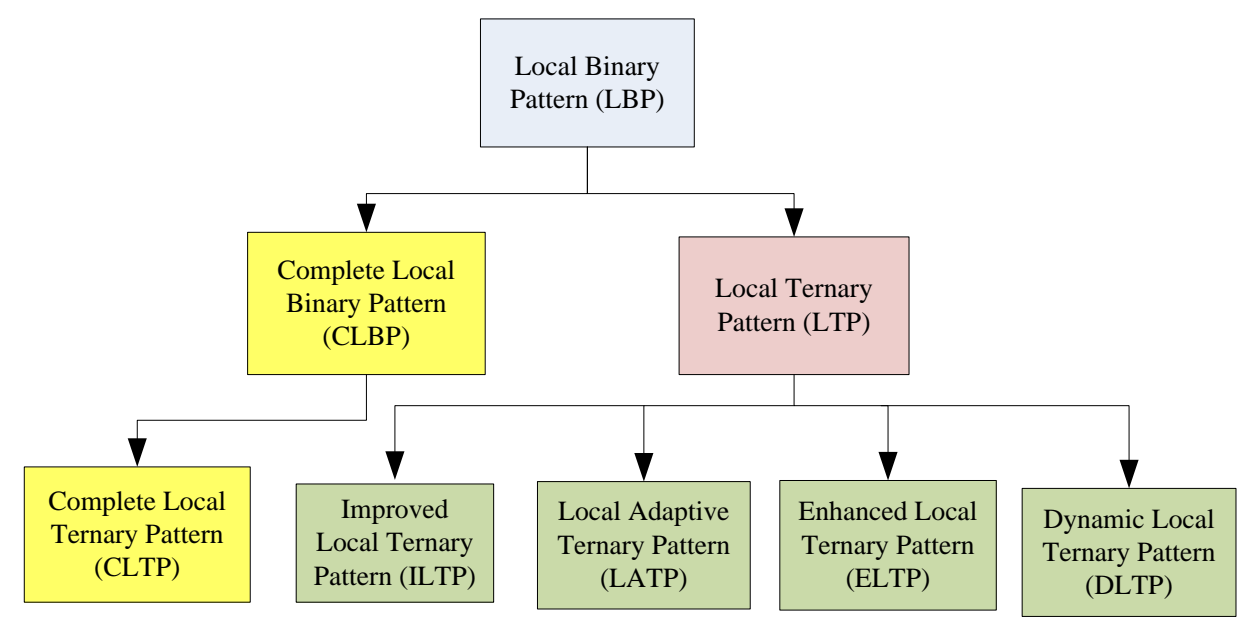

Figure 3:- Tree diagram of LBP and its extended versions

\section{Limitations of LBP and its extended variants:-}

When we are talking about texture descriptors, on one hand, expect to find only one approach that have capabilities to handle so many different problems that we are dealing in different applications such as illumination changes, rotation, scaling and bur problems. On second hand, the descriptors must be simple in computation and smaller in size so to avoid memory and processing time limitations.

Usually the LBP code is generated by computing two values in each of the pattern that is central pixel which normally used as a threshold and other one is neighborhood pixels which are the gray values around the center pixel value in the patch size.

Table 1:- The limitations and advantages of LBP and its extended techniques

\begin{tabular}{|l|l|l|}
\hline Texture descriptor & pros & cons \\
\hline LBP & Simple and low in complexity & Noise sensitive \\
\hline LTP & Robust to noise & Difficult to set absolute threshold value \\
\hline ILTP & $\begin{array}{l}\text { Robust to noise and automatic adoption of } \\
\text { threshold value }\end{array}$ & Only considered local features \\
\hline LATP & $\begin{array}{l}\text { Robust to noise and automatic adoption of } \\
\text { threshold value }\end{array}$ & Only considered local features \\
\hline $\begin{array}{l}\text { DLTP } \\
\text { ELTP }\end{array}$ & $\begin{array}{l}\text { Robust to noise and automatic adoption of } \\
\text { threshold value }\end{array}$ & Global features are neglected \\
\hline CLBP & $\begin{array}{l}\text { Considered global features } \\
\text { nonsidered global features and robust to }\end{array}$ & Difficult to set absolute threshold value \\
\hline noise &
\end{tabular}

One of main limitation in LBP descriptor as appeared that LBP can in the image. The reason behind this is that LBP is very sensitive to the noise with the central pixel as threshold value.

Same limitation comes in LBP variants such as Complete Local Binary Pattern CLBP. To overcome this weakness of LBP, Local Ternary Pattern is the first effort by introducing manual threshold value, which proves itself robust to noise with central pixel. But it is very challengeable task to set the right threshold value for particular application in order to get high performance. Another prominent issue with LTP threshold value comes out in a case when threshold value becomes exactly the same as of the difference of central pixel and neighbor hood pixel. In this case the outcome of LBP code becomes zero. To deal with this issue, recently noticeable extensions of LTP known as Improved Local Ternary Pattern (ILTP), Local Adaptive Ternary Pattern (LATP), Enhanced Local Ternary Pattern and Dynamic Local Ternary Pattern has been proposed, which are based on some automatic phenomena to set threshold value for every region. 
Secondly, it is clear from the literature that most of the LBP and LTP and their variant descriptor are based on its local features. Sometimes the information generated from the local features is not sufficient for classifying the patterns. Because of neglecting the global intensities of the images, sometimes texture descriptor cannot generate distinguish features between two different gray value based patches of the image.

The little effort towards this issue proposed in Complete Local binary Pattern (CLBP) and Complete Local Ternary Pattern (CLTP) to consider the global features for getting more accurate information about the patterns.

The advantages and limitations of Local Binary Pattern (LBP) and its extended versions are summarized in Table 1 and graphical directing these pros and cons in Figure 4.

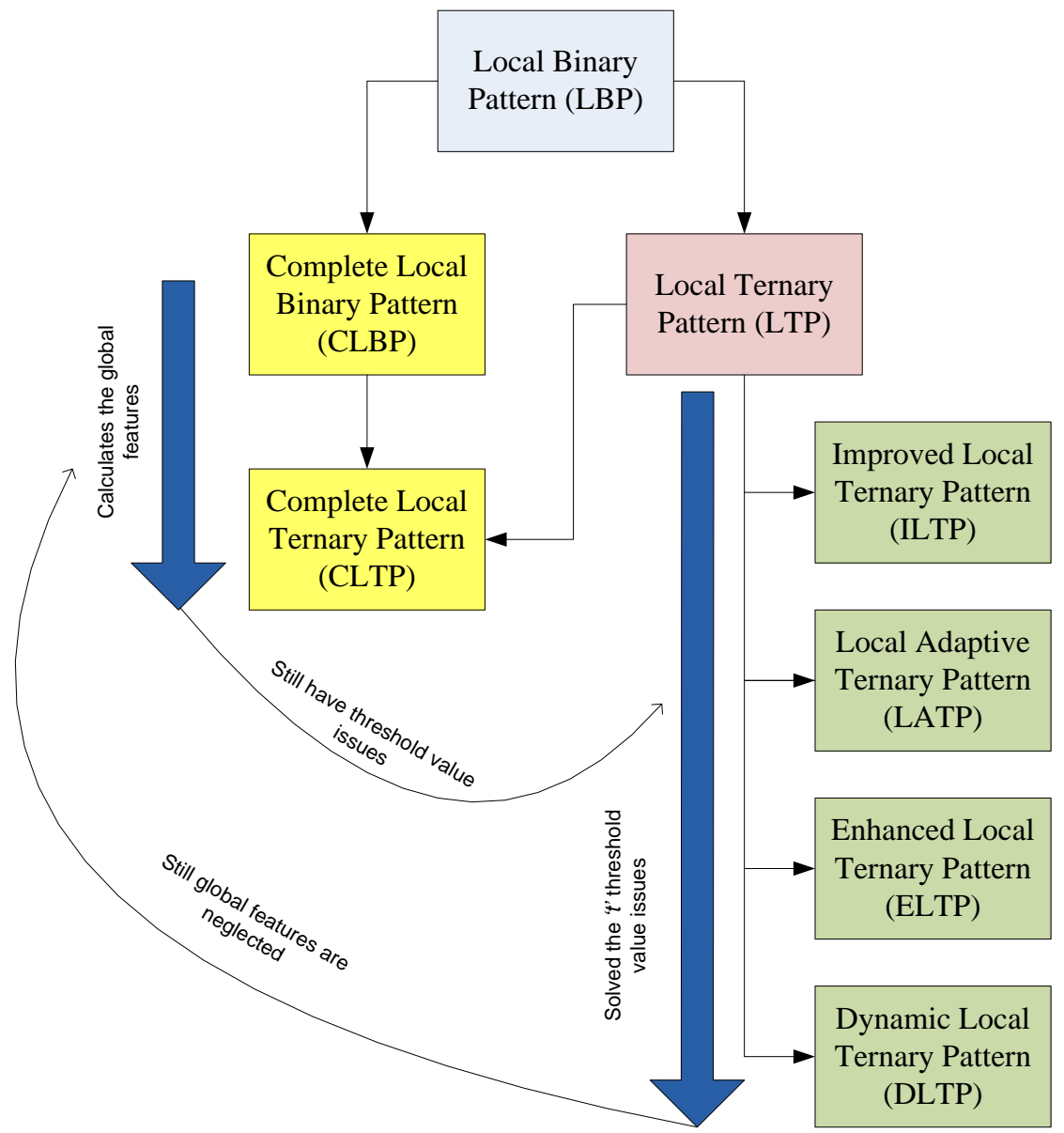

Fig 4:- Advantages and Disadvantages of LBP and its variants

\section{Conclusion:-}

In this review paper, it is conclude that, unfortunately, from the stat-of-art there is no stand-alone descriptor that covers the problems as already mentioned in this artical such as illumination changes, rotation, scaling and bur in every aspect. We already have been discussed the traditional LBP operator and its use of in number of different pattern recognition based. But still there are some limitations with LBP and its variant descriptors.

From the literature it is found that no single descriptor has been developed which covers all the limitations. Therefore, there is a need of a new robust texture descriptor that overcome the gapes and retains the advantages of reported descriptors. 


\section{References:-}

1. Chaudhuri, B. B., N. Sarkar, and P. Kundu. "Improved fractal geometry based texture segmentation technique." IEE Proceedings E (Computers and Digital Techniques) 140.5 (1993): 233-242.

2. Zhang, Jianguo, and Tieniu Tan. "Brief review of invariant texture analysis methods." Pattern recognition 35.3 (2002): 735-747.

3. Materka, Andrzej, and Michal Strzelecki. "Texture analysis methods-a review." Technical university of lodz, institute of electronics, COST B11 report, Brussels (1998): 9-11.

4. M"aenp"a"a T \& Pietik"ainen M (2005) Texture analysis with local binary patterns. Proc. C. Chen and P. Wang (eds.) Handbook of Pattern Recognition and Vision, World Scientific, Signapore, 3rd Edition, 197-216.

5. He, D., Wang, L.: Texture unit, texture spectrum and texture analysis. In: Geoscience and Remote Sensing, Symposium (1989)

6. Ojala, T., Pietikainen, M., Harwood, D.: Performance evaluation of texture measures with classification based on kullback discrimination of distributions. In: 12th IAPR International Conference (1994).

7. Ojala, T., Pietikäinen, M., Mäenpää, T.: Gray scale and rotation invariant texture classification with local binary patterns. In: European Conference on Computer Vision. Lecture Notes in Computer Science, vol. 1842, pp. 404-420. Springer, Berlin (2000)

8. Ojala, T., Pietikäinen, M., Harwood, D.: A comparative study of texture measures with classification based on feature distributions. Pattern Recognit. 29(1), 51-59 (1996)

9. Ojala T, Pietik"ainen M \& M"aenp“a"a T (2002b) Multiresolution gray-scale and rotation invariant texture classification with local binary patterns. IEEE Transactions on Pattern Analysis and Machine Intelligence 24(7): 971-987.

10. Pietikä Pietikainen, M., Hadid, A., Zhao, G., Ahonen, T.: Computer Vision Using Local Binary Patterns. Springer, London (2011)

11. Guo, Z., Zhang, L., Zhang, D.: A completed modeling of local binary pattern operator for texture classification. EE Trans. Image Process. vol. ePub (2010)

12. Tan, X., Triggs, B.: Enhanced local texture feature sets for face recognition under difficult lighting conditions. Anal. Model. Faces Gestures, LNCS 4778, 168-182 (2007)

13. Rassem, Taha H., and Bee Ee Khoo. "Completed local ternary pattern for rotation invariant texture classification." The Scientific World Journal 2014 (2014).

14. Yang, Wankou, and Changyin Sun. "Face recognition using improved local texture patterns." Intelligent Control and Automation (WCICA), 2011 9th World Congress on. IEEE, 2011.

15. Akhloufi, Moulay, and Abdelhakim Bendada. "Locally adaptive texture features for multispectral face recognition." Systems Man and Cybernetics (SMC), 2010 IEEE International Conference on. IEEE, 2010.

16. Ibrahim, Mohammad, Md Iftekharul Alam Efat, Humayun Kayesh Shamol, Shah Mostafa Khaled, Mohammad Shoyaib, and M. Abdullah-Al-Wadud.(2014).Dynamic Local Ternary Pattern for Face Recognition and Verification.In International Conference on Computer Engineering and Applications, no. 8. 10-12 janary, Tenerife, Spain.

17. Zhenyu, Wang, et al. "An enhanced Local Ternary Patterns method for face recognition." Control Conference (CCC), 2014 33rd Chinese. IEEE, 28-30 July, 2014, Nanjing, China 